\title{
Polyamide from lactams by reactive rotational molding via anionic ring-opening polymerization: Optimization of processing parameters
}

\author{
N. Barhoumi ${ }^{1}$, A. Maazouz ${ }^{1 *}$, M. Jaziri ${ }^{2}$, R. Abdelhedi ${ }^{2}$ \\ ${ }^{1}$ Ingénierie des Matériaux Polymères-(IMP/LMM), UMR-CNRS 5223, INSA-Lyon, Université de Lyon, 69621 Lyon, \\ 17 Avenue Jean Capelle, Villeurbanne Cedex, France \\ ${ }^{2}$ Laboratoire d'Electrochimie et Environnement. ENIS- Sfax, 3038 Sfax, Tunisie
}

Received 17 June 2012; accepted in revised form 1 September 2012

\begin{abstract}
A reactive rotational molding (RRM) process was developed to obtain a PA6 by activated anionic ring-opening polymerization of epsilon-caprolactam (APA6). Sodium caprolactamate (C10) and caprolactam magnesium bromide (C1) were employed as catalysts, and difunctional hexamethylene-1,6-dicarbamoylcaprolactam (C20) was used as an activator. The kinetics of the anionic polymerization of $\varepsilon$-caprolactam into polyamide 6 was monitored through dynamic rheology and differential scanning calorimetry measurements. The effect of the processing parameters, such as the polymerization temperature, different catalyst/activator combinations and concentrations, on the kinetics of polymerization is discussed. A temperature of $150^{\circ} \mathrm{C}$ was demonstrated to be the most appropriate. It was also found that crystallization may occur during PA6 polymerization and that the combination $\mathrm{C} 1 / \mathrm{C} 20$ was well suited as it permitted a suitable induction time. Isoviscosity curves were drawn in order to determine the available processing window for RRM. The properties of the obtained APA6 were compared with those of a conventionally rotomolded PA6. Results pointed at lower cycle times and increased tensile properties at weak deformation.
\end{abstract}

Keywords: processing technologies, anionic polymerization, $\varepsilon$-caprolactam, viscosity, reactive rotational molding

\section{Introduction}

Rotational molding has been regarded as a polymer processing technique with great potential [1]. The obtained products are virtually stress-free and without weld lines. Moreover there is no material waste, and the required molds are relatively inexpensive. Yet, its widespread growth is hindered by long production cycle times as well as by the fact that one can only use dry powders in order to meet material property demands. This is particularly true for polyethylene, which has limited properties. Several high performance resins, such as polyamides and polycarbonates, are also available to rotomolders offering better properties, but at a cost.
One way to overcome issues involved in the rotational molding of powders as well as gain engineering material properties is to utilize liquid resins. Immediate advantages include a reduced cycle time, an excellent reproduction of surface detail and a wider range of material properties. Based on these strong points, the use of liquid resin systems when performing rotational molding would offer numerous manufacturing benefits over the conventional powder process [2].

Additionally, the use of the RRM process renders possible the synthesis of engineering thermoplastics, e.g., polyamide 6 (PA6), with high molecular weights. In the case of PA6, this is done through

\footnotetext{
${ }^{*}$ Corresponding author, e-mail: Abderrahim.maazouz@insa-lyon.fr (C) BME-PT
} 
anionic polymerization of caprolactam by the aid of chain initiators and catalysts [3].

It is well known that anionic polymerization of $\varepsilon$ CL (APCL) occurs faster (i.e., over a few minutes) than classical hydrolytic polymerization, which takes about 12-24 h [4]. A fast polymerization process in addition to good mechanical properties of PA6 results in a wide range of applications for this polymer in reaction injection molding $[5,6]$, rotational molding $[1,7]$ and centrifugal molding [8-9]. This makes PA6 one of the main engineering materials in use today.

According to different reports [10-17], there are three main parameters that govern the polymerization of APCL: the catalyst (type and concentration), activator (type and concentration), and also the initial polymerization temperature. In almost all published studies, this process has been carried out in an inert atmosphere.

There are a number of problems that are inherent to the processing of reactive monomers and that limit the development and uptake of the process in industry [18]. These problems, which include difficulties in controlling the reaction viscosity and an uneven distribution of material, coupled with a lack of research in this area, have kept the use of reactive monomer systems in the rotational molding industry to a minimum [1]. Thus, one needs to gain fundamental understanding of the RRM of $\varepsilon$-caprolactam in order to determine a means to control its viscosity during anionic polymerization which in turn would facilitate industrial applications. Although the feasibility of the reaction has been known for a long time, there is still only limited industrial exploitation [19].

The influence of polymerization conditions (i.e., catalyst and activator type and also catalyst/activator combination) on the rate of polymerization is reported in the literature [14, 20, 21]. However, the choice of polymerizations conditions for the RRM process has yet to be described and optimized. As a continuation of previous investigations [1,2], which report on the possibility of reactive rotomolding of defect-free Nyrim ${ }^{\circledR}$ parts, our study presents a complementary approach based on determining the processing parameters required to obtain a polyamide 6 through reactive rotational molding by anionic polymerization of $\varepsilon$-caprolactam (APA6). The reactive mixture (caprolactam, catalyst and activator) was injected into a mold and the PA6 was formed in-situ by the anionic polymerization of $\varepsilon$-caprolactam at the set temperature during rotational molding.

In a first step, the article discusses the influence of the concentration and combination of activator and initiator, as well as the effect of the polymerization temperature on the kinetics of polymerization. The aim was to choose a suitable APA6 formulation for RRM processing with shorter cycle times. Subsequently, we attempted to define the optimal conditions of rotational molding by constructing isoviscosity curves and to understand the reactive forming process by monitoring the viscosity. Finally, the process has been described by contrasting it with classical rotational molding, and as a result, the properties of APA6 and PA6 were also compared.

\section{Experimental}

\subsection{Materials}

\subsubsection{Monomer, activator and catalysts of anionic polyamide 6}

An anionic polymerization grade of the caprolactam monomer (AP-NYLON ${ }^{\circledR}$ Caprolactam) was used in this study (Figure 1) as it has a low moisture content $(<100 \mathrm{ppm})$. The monomer also has a low viscosity $\left(4.87 \mathrm{mPa} \cdot \mathrm{s}\right.$ at $\left.100^{\circ} \mathrm{C}\right)$ and a low melting point $\left(T_{\mathrm{m}}=69^{\circ} \mathrm{C}\right)$.

Brüggolen $\mathrm{C} 20$, a difunctional hexamethylene-1,6dicarbamoylcaprolactam $(2 \mathrm{~mol} / \mathrm{kg}$ concentration in caprolactam) was added as an activator in combination with Brüggolen $\mathrm{C} 10$, sodium caprolactamate ( $1 \mathrm{~mol} / \mathrm{kg}$ concentration in caprolactam) or Brüggolen $\mathrm{C} 1$, caprolactam magnesium bromide ( $1.4 \mathrm{~mol} / \mathrm{kg}$ concentration in caprolactam). The melt temperatures of $\mathrm{C} 20, \mathrm{C} 1$ and $\mathrm{C} 10$ were $60^{\circ} \mathrm{C}$ and the substances were used to control the speed and quality of the AP process.

All reactants were supplied by Bruggemann Chemical, Germany, in sealed polyethylene-lined aluminum drums and were used without further processing or purification. Since anionic polymerization of $\varepsilon$-caprolactam is very sensitive to moisture,

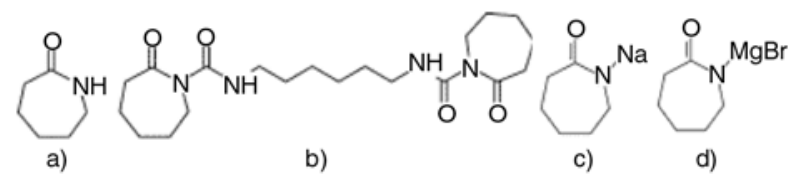

Figure 1. Chemical structures of (a) $\varepsilon$-caprolactam CL, (b) hexamethylene-1,6 dicarbamoylcaprolactam $\mathrm{C} 20$, (c) sodium caprolactamate C10, and (d) caprolactam magnesium bromide $\mathrm{C} 1$ [14] 


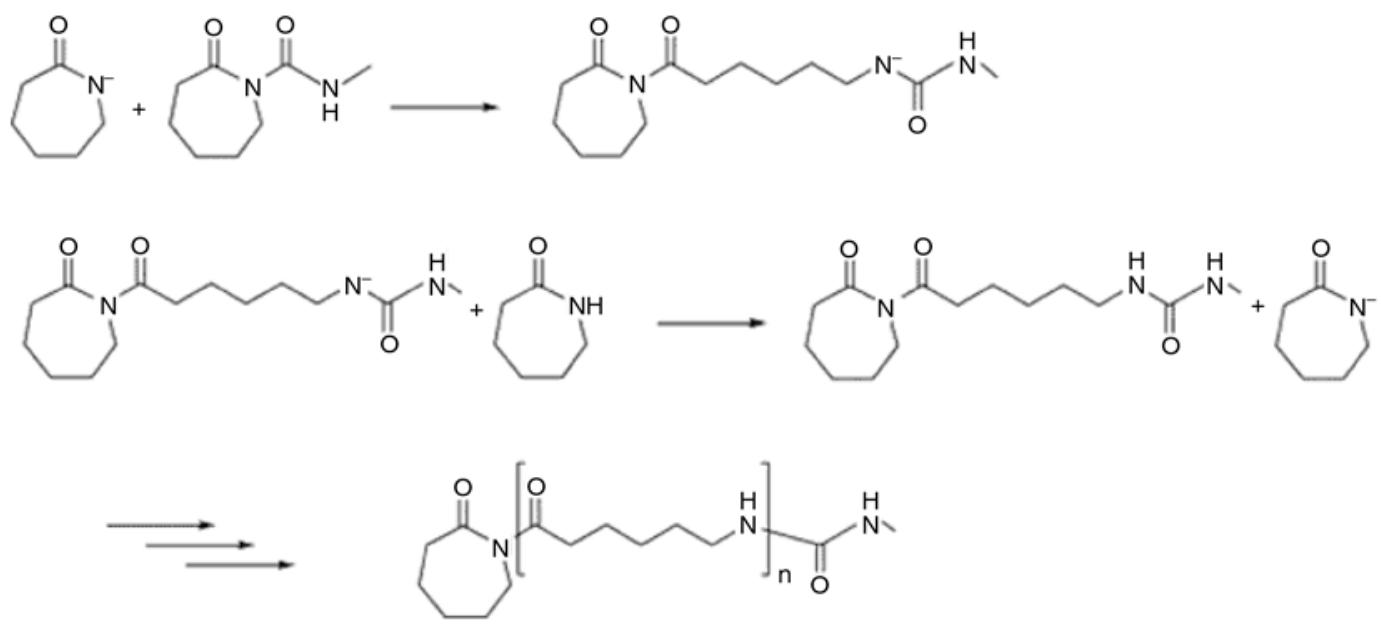

Figure 2. Anionic polymerization of $\varepsilon$-caprolactam into polyamide-6, using hexamethylene-1,6-dicarbamoylcaprolactam (C20) as the activator and caprolactam magnesium bromide (C1) as the initiator [14]

all the materials were dried overnight at $40^{\circ} \mathrm{C}$ under vacuum before use. The chemical structure of each material is presented in Figure 1, and the chemical pathway for the catalyst-activated $\varepsilon$-caprolactam reaction based on the $\mathrm{C} 1 / \mathrm{C} 20$ combination is shown in Figure 2 [14].

\subsubsection{PA6 (capron) obtained with the classical rotational molding process}

The PA6 used in this work is a commercial product referenced as Capron ${ }^{\circledR}$ RXA1482RO HS and supplied by BASF (France). The resin was available in powder form. Its number average molar mass was $34000 \mathrm{~g} / \mathrm{mol}$, and the melting point of this PA6 grade was $224^{\circ} \mathrm{C}$.

\subsection{In-situ polymerization of \&-caprolactam}

\subsubsection{Premix preparation}

The viscosity of APA6 is a function of the concentration of catalyst and initiator, as well as of temperature and time. Various formulations were investigated. The premix was charged to a reaction vessel and heated in an oil bath at $80^{\circ} \mathrm{C}$ under a nitrogen atmosphere: the monomer/activator-mixture was thus melted under stirring. In a second vessel, the catalyst was melted with the caprolactam. Subsequently, both mixtures were blended together and immediately characterized by DSC and with a rheometer in order to monitor in situ the anionic polymerization of $\varepsilon$-caprolactam. The various premixes reported in Table 1 in different mass ratio were denoted $\mathrm{CL} / \mathrm{CX} / \mathrm{CY}(\%$ caprolactam $/ \%$ catalyst $/ \%$ activator).

\subsubsection{Polymerization kinetics (chemorheology, DSC)}

The polymerization kinetics was monitored through isothermal measurements of the reaction exotherm with a differential scanning calorimeter (DSC Q10, TA Instruments, USA) and an ARES rheometer (Rheometeric Scientist, USA) used as a chemical reactor. The temperatures and compositions were varied with the aim of determining the process parameters.

In the case of the rheological analysis, the premix was shaken and quickly introduced with a syringe into the $(0.06) \mathrm{mm}$ gap set between the preheated cone and plate geometry at the polymerization temperature. The plate had a diameter of $40 \mathrm{~mm}$. Thus, the exchange surface between the polymerization medium and the area was small in comparison to the volume, thereby limiting the loss of premix by evaporation. The variations in complex viscosity during the polymerization step were then monitored by time sweep oscillatory experiments under a shear stress of $0.05 \mathrm{~Pa}$ and an angular frequency of

Table 1. Designations and compositions of premixes

\begin{tabular}{|l|c|c|c|c|}
\hline \multicolumn{1}{|c|}{ Premix } & $\begin{array}{c}\text { Mass ratio CL } \\
{[\mathbf{g}]}\end{array}$ & $\begin{array}{c}\text { Mass ratio C1 } \\
{[\mathbf{g}]}\end{array}$ & $\begin{array}{c}\text { Mass ratio C10 } \\
{[\mathbf{g}]}\end{array}$ & $\begin{array}{c}\text { Mass ratio C20 } \\
{[\mathbf{g}]}\end{array}$ \\
\hline $100 / 3 / 3(\mathrm{CL} / \mathrm{C} 1 / \mathrm{C} 20)$ & 100 & 3 & & 3 \\
\hline $100 / 3 / 3(\mathrm{CL} / \mathrm{C} 10 / \mathrm{C} 20)$ & 100 & & 3 & 3 \\
\hline $100 / 4 / 4(\mathrm{CL} / \mathrm{C} 1 / \mathrm{C} 20)$ & 100 & 4 & & 4 \\
\hline
\end{tabular}


$1 \mathrm{rad} / \mathrm{s}$. Nitrogen gas was used to prevent thermal oxidation.

The objective of the isothermal DSC analysis was to measure the reaction of polymerization heat flow versus time at several constant temperatures. The activator and catalyst were added to the molten monomer just above its melting point and this mixture was then quenched in nitrogen liquid to stop the reaction.

\subsubsection{Reactive rotational molding process of APA6}

A Rotoline pilot-scale shuttle-type rotational molding machine with an aluminum cube mold and a volume of $25 \times 25 \times 10 \mathrm{~cm}^{3}$ was used to produce the PA6 parts. The Datapaq ${ }^{\circledR}$ Tracker Telemetry system was utilized to measure, in real time, the temperatures of the oven, the external wall of the mold, and the internal air/nitrogen during the processing cycle. The reactive premixes were poured in the mold through an air vent, after which the mold's rotation and the thermal cycle were started. The rotation speeds of the two axes were 4 and $5 \mathrm{rpm}$.

The typical chemorheology of a reactive liquid system is shown in Figure 3 [2]. It includes four main steps, and for each regime a certain viscosity is required. At the start of the process, the viscosity needs to be relatively low so that the rotating pool mixes the ingredients and only deposits a thin film of reactive resin on the mold surface. As the processing time progresses, the viscosity should slowly rise so that the cascading flows distribute a thicker layer evenly over the mold. In the last flow phase, rimming finalizes the even distribution of resin on

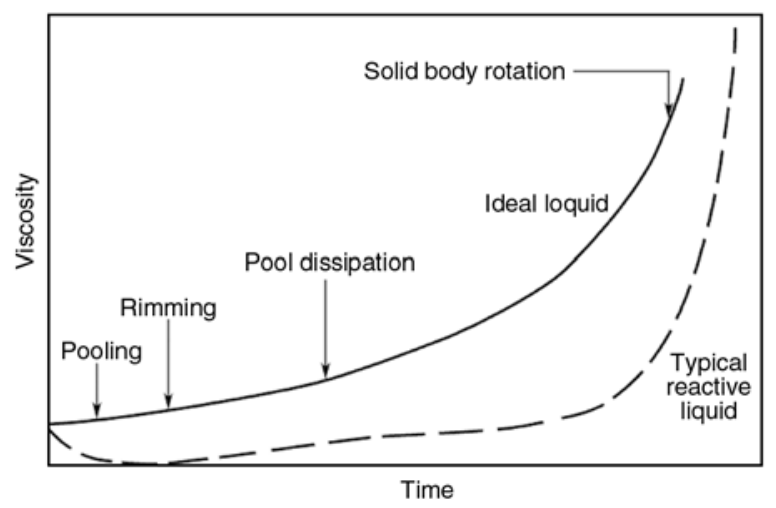

Figure 3. Typical variation of viscosity with curing time for a reactive liquid compared with an ideal flow behavior [22] (Redrawn from Figure 6.39 of Crawford and Throne with modification) the mold until gelation occurs, resulting in solid body rotation.

\subsection{Characterization methods}

\subsubsection{Differential scanning calorimetry (DSC)}

Differential scanning calorimetry (DSC Q10, TA Instruments, USA) was performed to investigate the crystallization and melting behaviors of the APA6 samples. Specimens with weights of 5-10 mg were cut for the measurement. Thermograms were recorded in three consecutive runs: (1) a first heating run, from room temperature to $240^{\circ} \mathrm{C}$, followed by (2) cooling, from 240 to $25^{\circ} \mathrm{C}$ and finally (3) a second heating run, from 25 to $240^{\circ} \mathrm{C}$. All experiments were performed at a heating/cooling rate of $10^{\circ} \mathrm{C} / \mathrm{min}$ under an argon atmosphere to avoid thermal degradation. The degree of crystallisation, $\chi_{\mathrm{c}}$, was calculated considering a melting enthalpy of $190 \mathrm{~J} / \mathrm{g}$ for a $100 \%$ crystalline polyamide 6 [17].

The polymerization was exothermic and the experiments involved measuring the reaction heat flow versus time at several constant temperatures. The activator and catalyst were added to the molten monomer just above its melting point after which the mixture was quenched in liquid nitrogen to stop the reaction. The cold (crystallized) mixture was then sealed in DSC capsules under a dry and oxygen-free atmosphere.

\subsubsection{Monomer conversion}

The degrees of conversion $(X)$ of the APA6 samples were determined for various resin formulations at several temperatures. The polymerized samples were ground, weighed $\left(m_{\text {tot }}\right)$ and refluxed overnight in demineralized water. After drying, the samples were weighed again $\left(m_{\text {pol }}\right)$. While the caprolactam monomer dissolved easily in water, the polyamide6 did not and the degree of conversion was consequently determined according to Equation (1) [23]:

$X=\frac{m_{\mathrm{pol}}}{m_{\mathrm{tot}}} \cdot 100 \%$

\subsubsection{The viscometric weight-average molecular weight}

The molecular weight $\left(M_{\mathrm{w}}\right)$ of the polyamides was determined by intrinsic viscosity measurements using formic acid as solvent at a concentration of $0.2 \mathrm{~g} / \mathrm{dL}$ with a suspended-level Ubbelohde viscometer at $25^{\circ} \mathrm{C}$. Based on the results from the 
intrinsic viscosity measurements $(\eta), M_{\mathrm{w}}$ could be determined according to Equation (2) [5]:

$M_{\mathrm{W}}=2.81 \cdot 10^{4} \eta^{1.35}$

\subsubsection{Mechanical characterization}

The tensile specimens were obtained with a tensile Sample Cutting Die from rotomolded parts in press at $80^{\circ} \mathrm{C}$.

Tensile tests were carried out using a conventional Instron tensile machine at room temperature according to ASTM D368M test, with a crosshead speed of $10 \mathrm{~mm} / \mathrm{min}$. At least five specimens were tested for each material.

\section{Results and discussion}

\subsection{Optimizing polymerization conditions for the RRM process}

3.1.1. Choice of activator - catalyst combination

The polymerization reaction consists of three steps: (i) anion formation, (ii) complex formation between the catalyst and the activator and (iii) polymerization through the anions during which an anion is regenerated after addition of a monomer [20].

Figure 4 shows the change in complex viscosity during the APCL in the presence of two activator-catalyst combinations $(\mathrm{C} 20-\mathrm{C} 1$ and $\mathrm{C} 20-\mathrm{C} 10)$ polymerized at $160^{\circ} \mathrm{C}$. The polymerization seemed to get off to a slow start when the $\mathrm{C} 1$ catalyst was used, as proven by the viscosity remaining constant at short times since no complex was formed initially [23].

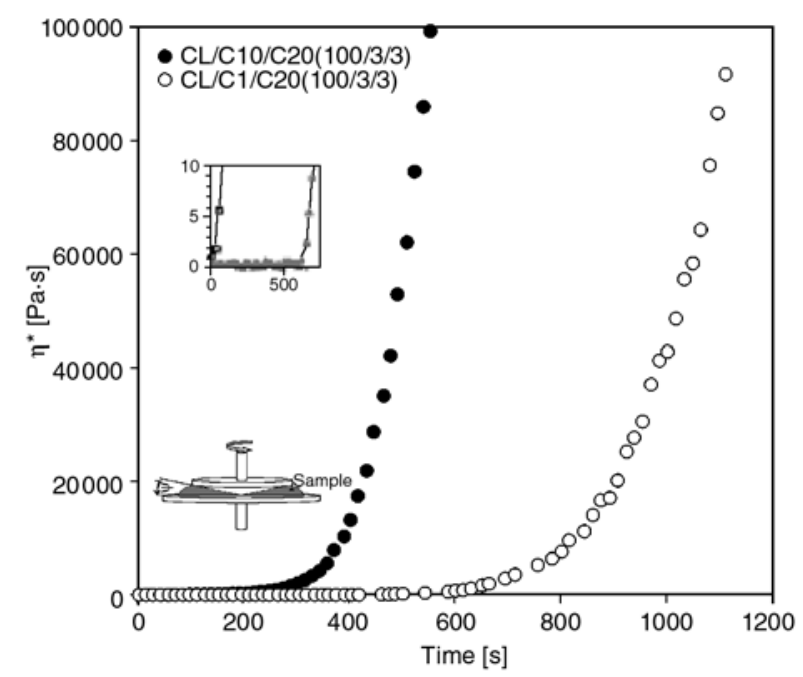

Figure 4. In situ polymerization of $\varepsilon$-caprolactam between the plates of the rheometer at $T=160^{\circ} \mathrm{C}$ for two activator- catalyst combination
However, after a single monomer addition, the carbamoylcaprolactam group was replaced by an acetylcaprolactam group. Since the latter was able to form a complex with the initiator, the reaction rate increased after a while.

In the case where catalyst $\mathrm{C} 10$ was used, there was no induction period for the AAPCL. This behavior was due to the complex formation between the catalyst $\mathrm{C} 10$ and the activator $\mathrm{C} 20$ [23]. It could thus be concluded that the $\mathrm{C} 20-\mathrm{C} 10$ formulation was unsuitable for the RRM process due to the reaction being immediate and the liquid not having enough time to spread before gelation, causing defects to be generated. As an alternative, the slower reacting formulation, that is $\mathrm{C} 20-\mathrm{C} 1$, could provide the required time window for the RRM process. As can be seen in Figure 4, there was an induction time of 6 minutes before the start of polymerization.

\subsubsection{Effect of polymerization temperature and activator-catalyst concentration}

Figure 5 shows that the final steady-state value of the complex viscosity did not seem to depend on the temperature, but rather on the time of the polymerization reaction. It can be noted that the induction time decreased with increasing temperature and concentration of $\mathrm{C} 1 / \mathrm{C} 20$. Besides the type of activator and initiator used, also the amount of these reactive species and the temperature influenced the reaction [23]. It was shown that the concentration and polymerization temperature increased the polymerization rate.

A higher concentration of the activator increased the amount of initiator points for chain growth [23]. Also, with a higher concentration of catalyst, more

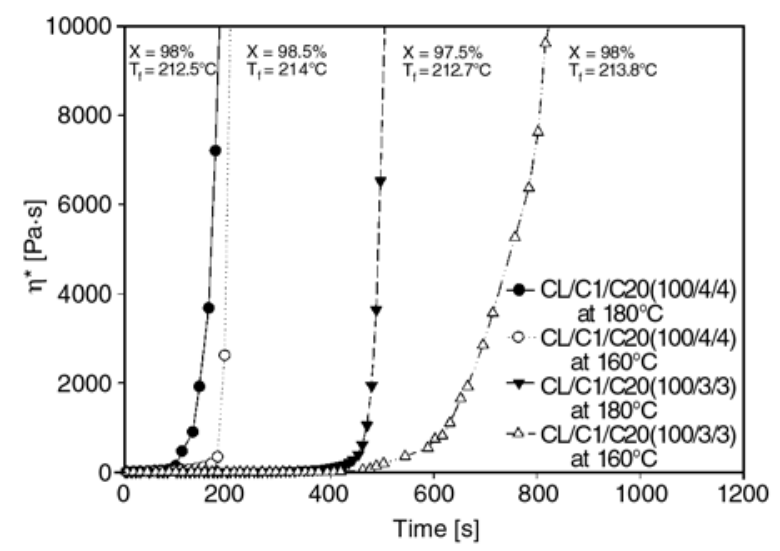

Figure 5. Effect of the polymerization temperature and concentration on the viscosity time profile of $\mathrm{CL} / \mathrm{C} 1 /$ $\mathrm{C} 20$ 
anions were set free and more complexes could be formed. As a consequence, the polymerization rate went up.

\section{In-situ monitoring of polymerization and crystallization by rheology and DSC}

The monitoring of the change in crystallinity of the APA6 material during the reaction is shown in Figures 6 and 7. By combining the curves obtained from isothermal DSC and rheological measurements, it could be concluded that:

At lower temperatures $\left(150-160^{\circ} \mathrm{C}\right)$, the crystallization occurred faster and the heat flow pointed at the polymerization and crystallization taking place simultaneously and not being separated in this temperature range. This simultaneous process can affect the crystal morphology and enhance the final properties of the polymer.

At higher temperature $\left(170-180^{\circ} \mathrm{C}\right)$, as seen in Figure 7 , the crystallization began after the polymerization isotherm was completed. If the polymerization and crystallization were consecutive, i.e., if the molecular weight build-up was completed before the start of crystallization, the crystallization kinetics would not differ from the melt-crystallized PA6 except for a time-shift equal to the time needed for polymerization. Since the polymerization took place below the final polymer melting and crystallization points $\left(T_{\mathrm{m}}=224^{\circ} \mathrm{C}, T_{\mathrm{c}}=185^{\circ} \mathrm{C}\right)$, the crystallization became initiated during polymerization. In order to obtain optimal polymer properties, it was essential to balance the rate of polymerization and crystallization. The reaction temperature adversely affected both rates: with increasing temperature, the polymerization rate became higher, whereas the crystallization slowed down [23].

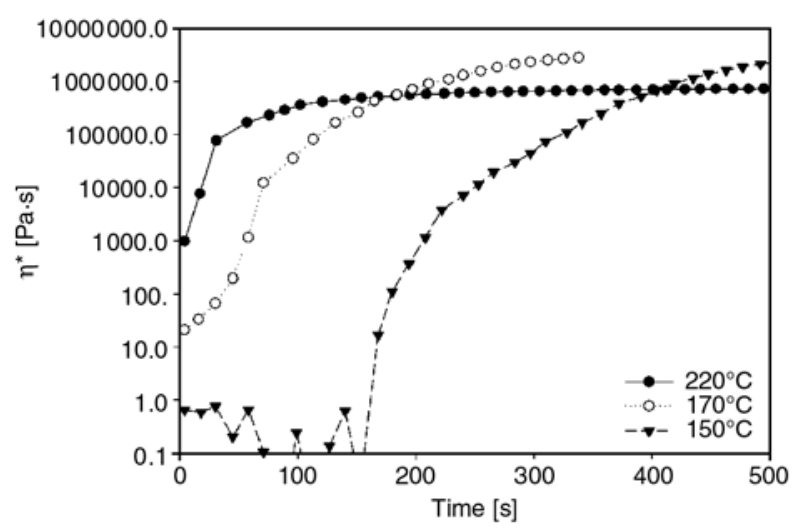

Figure 6. Viscosity-time profile of CL/C1/C20 (100/4/4) at different temperatures

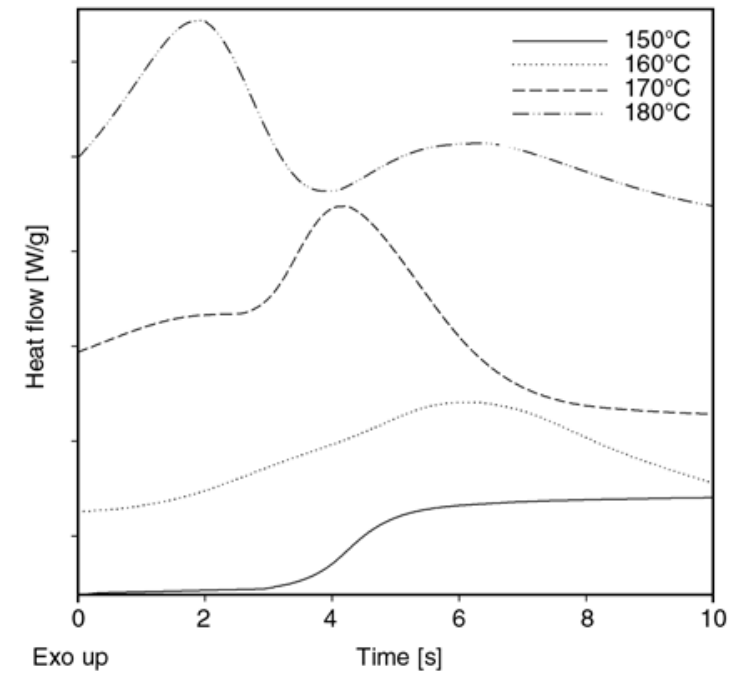

Figure 7. In-situ monitoring by DSC of the crystallization during isothermal polymerization of $\mathrm{CL} / \mathrm{C} 1 / \mathrm{C} 20$ $(100 / 4 / 4)$ at different temperatures

At $220^{\circ} \mathrm{C}$, only the PA6 polymerization occurs (the temperature is close to the PA6 melting temperature $\sim 224^{\circ} \mathrm{C}$. In this case, the viscosity increases during the build-up of the molecular weight.

When the reaction temperature was too low, the crystallization was too fast and reactive chain-ends and monomer could get trapped inside the crystals before being able to polymerize. As a result, the final obtained conversions were low. On the other hand, when the reaction temperature was too high, the growth rate of the chains was significant, whereas the formation of crystals became delayed. The higher the molecular weight, the more the polymer chains became entangled. As a consequence, the resistance encountered during crystal formation increased, which reduced the final degree of crystallinity [23].

\section{Influence of polymerization temperature on the polymer melting point}

Figures 8 and 9 show typical DSC thermograms of polymer melting and crystallization peaks at several polymerization temperatures. It can be seen that the polymer melting temperature decreased with an increase of the polymerization temperature. At polymerization temperatures below the final melting point, the crystallization occurred during the polymerization and it caused an increase of the maximum melting point. As a result, crystallites formed during polymerization which led to much higher melting temperatures.

When increasing the polymerization temperature from 160 to $180^{\circ} \mathrm{C}$, a drop in the polymer melting 
point of 2 to $6^{\circ} \mathrm{C}$ was observed, see Figure 8 and 9 . Three possible explanations can be given. First of all, residual caprolactam can reduce the polymer melting point [17]. Ricco et al. have reported melting point depressions between 3.5 and $5.8^{\circ} \mathrm{C}$ per wt $\%$ residual caprolactam [25]. Second, the increasing number of crystal imperfections induced by the formation of branch-points can cause a decrease in melting temperatures, simply because less energy is required in order to break down a crystal [26].

It is reported for APA6 that branching can even cause a transition in the crystal structure [25], which is the third reason for the lower melting points. This is explained as follows. Two types of crystals appear in anionic PA6, i.e., those with the $\alpha$-structure $\left(T_{\mathrm{m}}=\right.$ $\left.220^{\circ} \mathrm{C}\right)$ and those with the $\gamma$-structure $\left(T_{\mathrm{m}}=214^{\circ} \mathrm{C}\right)$. For anionic PA6, it is reported that due to excessive branching, the $\gamma$ content can increase [25], hence reducing the melting point.

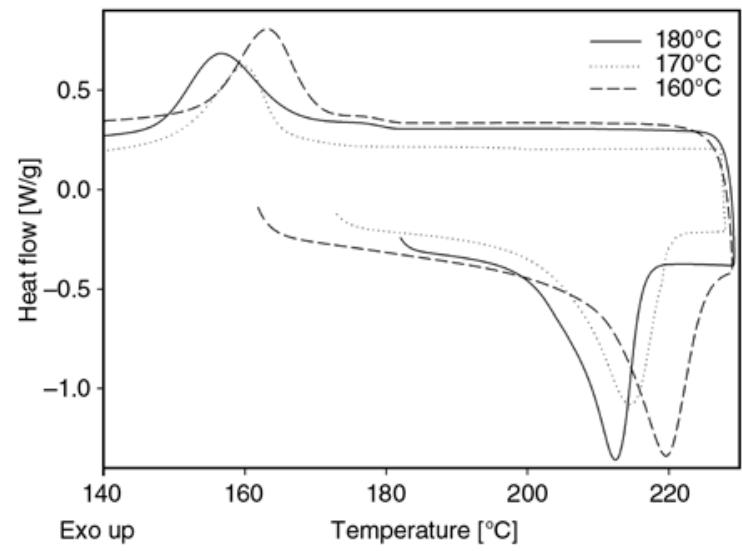

Figure 8. DSC thermograms of heating and cooling of CL/C1/C20 (100/4/4) for various polymerization temperatures after isothermal polymerization in DSC

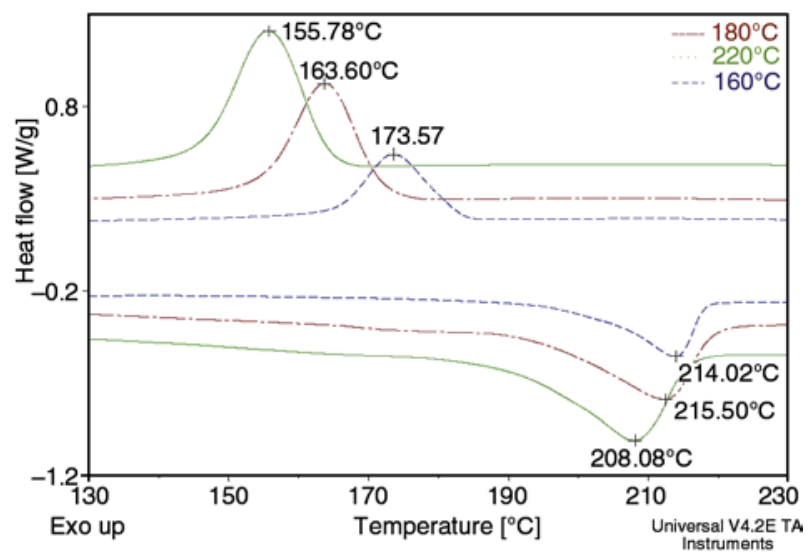

Figure 9. DSC thermogram of the cooling and second heating run for CL/C1/C20 (100/4/4) obtained with rheological tests at different temperatures after isothermal polymerization in the rheometer

\subsection{Processing}

\subsubsection{Chemorheology}

In the case of reactive rotational molding, the process is controlled by the variation in viscosity during polymerization [2]. Since the viscosity becomes increased as the polymerization progresses, a specific amount of time at a low viscosity is required to spread the liquid in the mold during rotation. It is therefore important to predict this available induction time, as it depends on the reaction kinetics and thus on the temperature.

Figures 10 and 11 present isoviscosity curves versus time and temperature of the two premixed blends CL/C1/C20 (100/3/3 and 100/4/4). The curves, obtained isothermally, range from 1 to $10^{5} \mathrm{~Pa} \cdot \mathrm{s}$. The one at $1 \mathrm{~Pa} \cdot \mathrm{s}$ renders it possible to determine the

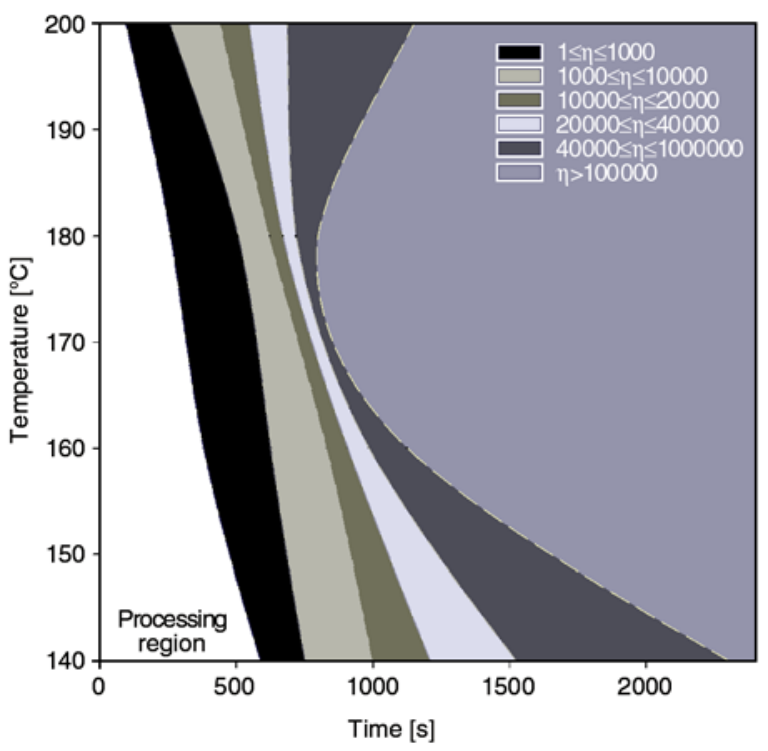

Figure 10. The full range of isoviscosities versus time and temperature during polymerization of $\mathrm{CL} / \mathrm{C} 1 /$ C20 (100/3/3)

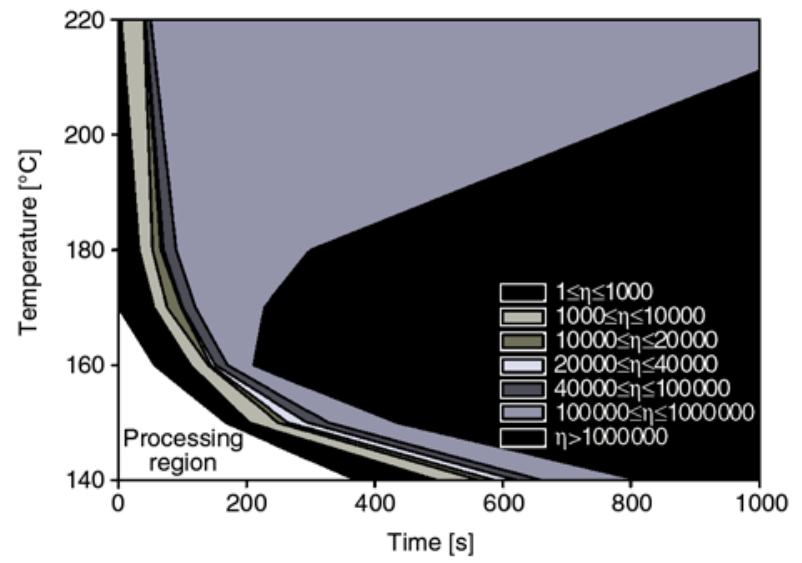

Figure 11. The full range of isoviscosities versus time and temperature during polymerization of $\mathrm{CL} / \mathrm{C} 1 /$ C20 (100/4/4) 
available induction time versus temperature, as reported by Crawford [18]. The ideal viscosity for rotational molding of liquids is between 0.05 and $1 \mathrm{~Pa} \cdot \mathrm{s}$. As the viscosity is dependent on temperature and the concentration of catalyst/activator, there was a minimum induction time for an isoviscosity curve at high temperature and concentration. Figure 10 and 11 portray the moldability zones, defining the areas where a successful rotational molding is expected.

Below the final polymer melting temperature and with short amounts of time, the viscosity increase was caused by the polymerization; at longer periods of time the viscosity increase was also due to the crystallization [19].

The choice of minimum and optimum cycle times requires a minimum temperature representing the cycle that uses the lowest energy in order to obtain the rotomolded APA6 part. Furthermore, it has been shown that a temperature of $150^{\circ} \mathrm{C}$ offers the highest tensile properties $[19,23,20]$. Therefore, based on the isoviscosity curves of the premix $\mathrm{CL} / \mathrm{C} 1 / \mathrm{C} 20$ $(100 / 4 / 4)$, we can expect the molding operation to be successfully carried out at this temperature with a short cycle time.

\subsubsection{Criterion for producing defect-free APA6 parts in a biaxially rotating mold}

In order to achieve a properly molded part, the material must be in the rubber state before gelation takes place [27]. The material reaction viscosity should increase slowly in the range within which solid body rotation occurs. However, if the initial viscosity is too low, the rate of increase will have no effect on the finished part [18].

Figure 12 shows the viscosity profile of the premix $\mathrm{CL} / \mathrm{C} 1 / \mathrm{C} 20(100 / 4 / 4)$ at $150^{\circ} \mathrm{C}$. From this profile,

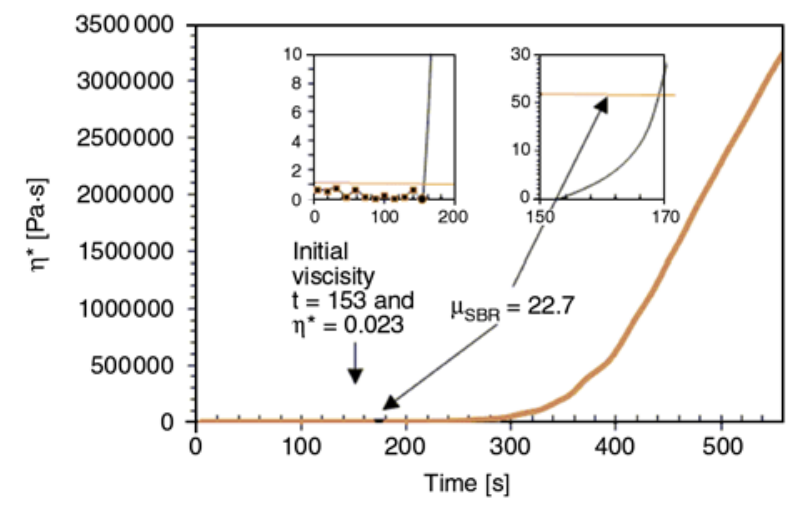

Figure 12. Viscosity-time profile of CL/C1/C20 (100/4/4) at $150^{\circ} \mathrm{C}$ the initial and minimum viscosity was determined and, as can be seen, there was sufficient induction time for the spreading.

The rotational speed of the primary $\left(W_{1}\right)$ and secondary $\left(W_{2}\right)$ axes was respectively 4 and $5 \mathrm{rpm}$ $(4 \cdot 2 \pi / 60$ and $5 \cdot 2 \pi / 60) \mathrm{rad} / \mathrm{s}$ in the rotational molding test. A lowest possible rotational speed should be employed to minimize bubble formation [2]. The material density was assumed to be $1100 \mathrm{~kg} / \mathrm{m}^{3}$. The wall thickness of the part was $0.002 \mathrm{~m}$ and the mold's major $\left(R_{1}\right)$ and minor radius $\left(R_{2}\right)$ : were 0.125 and $0.05 \mathrm{~m}$. The maximum solid-body rotation viscosity for the stated range of conditions was obtained according to Equation (3) as [2]:

$\mu_{\mathrm{SBR}}=\frac{0.7652 \rho d}{R \omega^{1.5}}$

where $\mu_{\mathrm{SBR}}=$ viscosity at which solid-body rotation occurs (Equation (4)), $p=$ fluid density, $d=$ finished wall part thickness, $W=$ angular rotation speed $\left(W=\left(W_{1}^{2}+W_{2}^{2}\right)^{1 / 2}\right)$, and $R$ cylinder radius $(R=$ $\left.\left(R_{1}^{2}+R_{2}^{2}\right)^{1 / 2}\right)$.

$$
\begin{aligned}
\mu_{\mathrm{SBR}} & =\frac{0.7652 \cdot 1100 \cdot 2 \cdot 10^{-3}}{\left(0.125^{2}+0.05^{2}\right)^{1 / 2} \cdot\left(\left(5 \cdot \frac{2 \pi}{60}\right)^{2}+\left(4 \cdot \frac{2 \pi}{60}\right)^{2}\right)^{1.5 / 2}}= \\
& =22.7 \mathrm{~Pa} \cdot \mathrm{s}
\end{aligned}
$$

Equation (3) predicts a viscosity of $22.7 \mathrm{~Pa} \cdot \mathrm{s}$ for solid body rotation, and this result is marked in Figure 12. It can be seen from this that the material was in the rubber state just before the viscosity increased rapidly to gelation.

In summary, based on the rheological and DSC test results, the formulation $\mathrm{CL} / \mathrm{C} 1 / \mathrm{C} 20(100 / 4 / 4)$ at $150^{\circ} \mathrm{C}$ was selected and was used to fabricate APA6 parts with the RRM process.

\subsection{Comparison between rotomolding PA6 parts obtained with RM and RRM \\ 3.3.1. Monitoring the process}

Temperatures profiles for the internal air, external mold and oven were recorded using thermocouples during the heating and cooling cycles of Capron. The processing conditions included: a weight charge of $450 \mathrm{~g}$, an oven temperature of $350^{\circ} \mathrm{C}$, a rotational speed of 4 and $5 \mathrm{rpm}$, and still air cooling. As, can be seen in Figure 13, the internal air temperature rose at a steady rate since it depended on the oven temperature. It exhibited four identifiable stages: induc- 


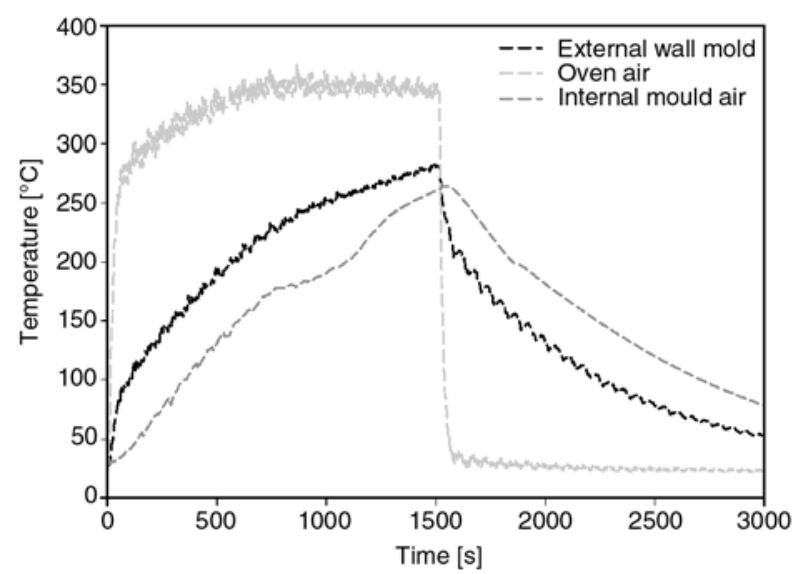

Figure 13. Temperature measurement in the oven and the mold during rotational molding of PA6 capron

tion, adherence of powder, melting-sintering of the powder and fusion-densification [28, 29], as proposed by Harkin-Jones and Crawford [2]. The experimental results in Figure 10 suggest a cycle time of 45 minute to obtain a rotomolded PA6 part. The procedure for making a biaxially and reactive rotational molded part was as follows. The mold was placed in the oven until the required mold temperature was achieved. The mold temperature was output onto a computer in real time via a Rotolog temperature measuring system. When the desired temperature was achieved, the mold was removed from the oven and rotated into the correct position for filling. The vent pipe was removed from the mold and the material was poured into the funnel, at which stage the mold was purged with dry nitrogen. The vent pipe was replaced as quickly as possible and the mold was put into rotation for the required cycle time. Finally the cooling cycle (if required) was started.

By combining the viscosity curve of the APA6 material (Figure 12) with the rotational motion of

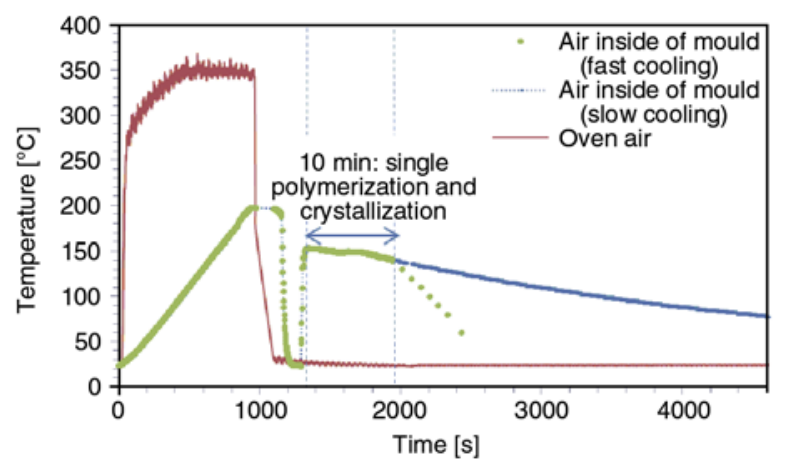

Figure 14. Temperature-time profiles for rotomolded anionic APA6: CL/C1/C20 (100/4/4) at $150^{\circ} \mathrm{C}$ polymerized out of the mold the mold, see Figure 14, it was determined that the reactive premix passed through several flow phases. Before the rotation started, the liquid formed a pool at the bottom of the mold. During rotation the liquid was lifted up the mold wall in the direction of the rotation $[2,4]$. The thickness of the layer is dependent on the linear velocity of rotation, the liquid viscosity and the liquid density. Based on the exothermic reaction of polymerization, as shown in Figure 12, it should be noted that the heat of melting resulted in the heating of the liquid, reduced the viscosity slightly during molding, before it started to increase again. Eventually, the viscosity of the liquid became sufficiently high for the mold wall to support the entire pool, resulting in solid body rotation. Ideally, solid body rotation should occur just before the viscosity of the plastic starts to increase rapidly as shown in Figure 12 [3].

\subsubsection{Characterization of PA6 obtained with RM and RRM}

This section compares the thermal and tensile properties of two polyamides:

- PA6: capron, processed by melt rotational molding (Figure 13).

- APA6: polymerized and rotomolded by reactive processing at a temperature of $150^{\circ} \mathrm{C}$ (Figure 14). Anionic polymerization at $150^{\circ} \mathrm{C}$ led to a similar degree of conversion as the melt-processed PA6 (see Table 2). However, in the case of the reactive process, the polymer chains had already attained a high molecular weight. Figure 15 presents typical DSC thermograms obtained for the final samples $\mathrm{CL} / \mathrm{C} 1 / \mathrm{C} 20(100 / 4 / 4)$ prepared at $150^{\circ} \mathrm{C}$ with $\mathrm{RRM}$ and commercial PA6 (Capron) obtained with the

Table 2. Material characteristics and processing parameters when comparing classical versus reactive processing

\begin{tabular}{|l|c|c|}
\hline $\begin{array}{c}\text { Rotational molding technique } \\
\text { of PA6 }\end{array}$ & Classical & Reactive \\
\hline Temperature & $T \sim 240^{\circ} \mathrm{C}$ & $T=150^{\circ} \mathrm{C}$ \\
\hline Cycle time & $t>40 \mathrm{~min}$ & $t=15-20 \mathrm{~min}$ \\
\hline Speed ratio $(\mathrm{S} 1 / \mathrm{S} 2)$ & $5 / 4$ & $5 / 4$ \\
\hline Melting point $T_{\mathrm{f}}\left[{ }^{\circ} \mathrm{C}\right]$ & 224.3 & 224 \\
\hline Degree of cristallinity [\%] & 28 & 49 \\
\hline Degree of conversion [\%] & $98.81 \%$ & $98.93 \%$ \\
\hline Intrinsic viscosity [dL/g] & 1.07 & 7 \\
\hline Molecular weight [g/mol] & 30778 & 182594 \\
\hline Tensile properties & 750 & 1560 \\
\hline Young's modulus [MPa] & 62 & 80 \\
\hline Yield stress [MPa] & 32 & 64 \\
\hline Elongation at break [\%] & \multicolumn{2}{|l}{} \\
\hline
\end{tabular}




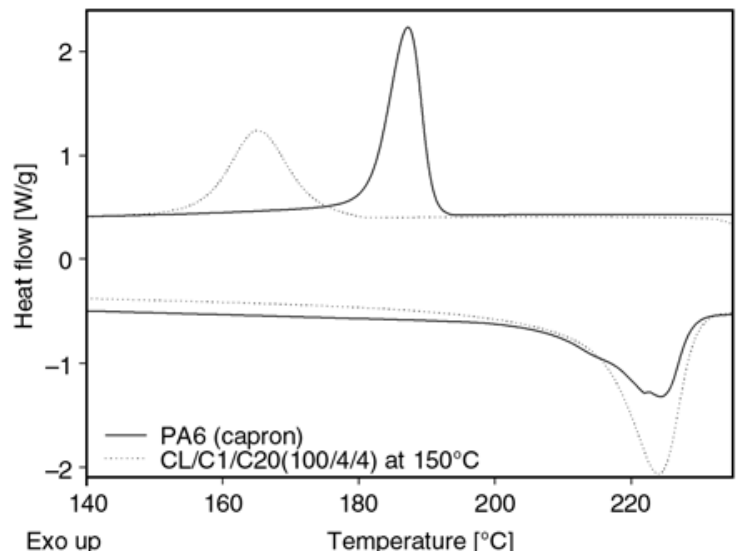

Figure 15. DSC traces of the cooling and second heating cycles of a APA6: Capron part obtained with rotational molding

melt process. A comparison of these results indicate that the melting temperature of this sample, i.e., $224^{\circ} \mathrm{C}$, is very close to that of PA6, i.e., $224.3^{\circ} \mathrm{C}$ (see Table 2) [15]. Nevertheless, as can be seen, the heat of melt of APA6 was greater than PA6, indicating a higher degree of crystallinity, is expected to lead to a higher modulus and tensile strength and to a lower elongation [10].

The initial crystallization should take place at approx. $150^{\circ} \mathrm{C}$. Since the polymerization occurred below the final polymer melting and crystallization points (i.e., $T_{\mathrm{m}}=224^{\circ} \mathrm{C}, T_{\mathrm{c}}=187^{\circ} \mathrm{C}$ ), the crystallization became initiated already during polymerization despite that this temperature was well below the crystallization temperature of PA6 $\left(T_{\mathrm{c}}=187^{\circ} \mathrm{C}\right)$. As a consequence, the crystal nuclei were plentiful and the final size of the spherulites was relatively small. Combining this with the high molecular weight of APA6 [22], one could expect that a single polymer chain was part of many of these small spherulites, which suggests a polymer morphology in which small spherulites were connected by a well-developed network of tie-molecules [23].

Tensile tests were performed on specimens obtained from the rotomolded parts. The typical stress-strain curves of PA6 and APA6 are shown in Figure 16, and a summary of the tensile properties, including Young's modulus $(E)$, tensile strength $\left(\sigma_{\mathrm{Se}}\right)$, and elongation at break $\left(A_{\mathrm{r}} \%\right)$, are listed in Table 2 . APA6 polymerized at $150{ }^{\circ} \mathrm{C}$ show a pronounced tensile modulus and yield strength when compared with the PA6 elaborated through melting. This was due to the significantly higher degree of crystallinity, cf. Figure 15, Table 2. Furthermore, the corresponding values of elongation at break (Table 2) were

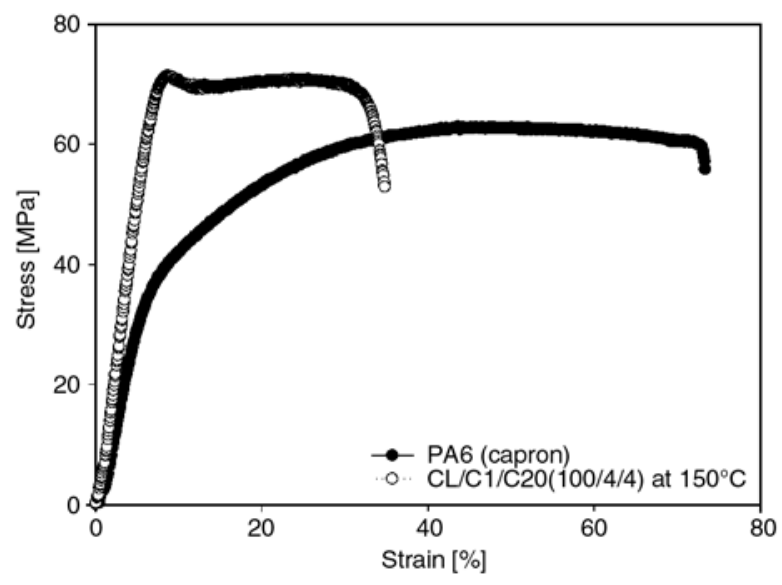

Figure 16. Typical stress-strain curves of PA6 Capron and APA6 at a crosshead speed of $10 \mathrm{~mm} / \mathrm{min}$

lower. This made APA6 less ductile as compared to the melt-processed capron.

It could therefore be concluded that, as with most semi-crystalline polymers, the mechanical properties of APA6 were predominantly determined by the degree of crystallinity and the level of conversion [14]. Furthermore, such polymerization-induced crystallization might be interesting as a means to decrease internal stresses [20] - a result that supports the studies of K. van Rijswijk et al. [14] and Udipi et al. [20], in which the highest tensile properties of APA6 at weak deformations were obtained at a mold temperature of $150^{\circ} \mathrm{C}$.

\section{Conclusions}

The kinetics of the APCL were investigated via DSC and rheology. The use of a rheometer as a chemical reactor rendered it possible to monitor the polymerization of $\varepsilon$-caprolactam.

In order to optimize the processing parameters for a successful rotational molding process, the effect of different activator-initiator concentrations and combinations, as well as of the polymerization temperature, was investigated. It was shown that, while the reaction mechanism was determined by the activator-initiator combination, the concentration and polymerization temperature had a large influence on the reaction rate and the physical properties of the polymer. The catalyst $\mathrm{C} 1$ was more suitable than its C10 counterpart as it rendered possible a suitable induction time. The formulation 100/4/4 at temperature of $150^{\circ} \mathrm{C}$ was thus suggested.

The isoviscosity curves helped us to limit the rotational molding domain. A material with a viscosity profile could thus be formulated and tested. Finally, the APA 6 obtained by reactive processing had a 
shorter cycle time and superior tensile properties at weak deformation as opposed to melt processed PA6.

\section{References}

[1] Harkin-Jones E., Crawford R. J.: Mechanical properties of rotationally molded nyrim. Polymer Engineering and Science, 36, 615-625 (1996).

DOI: $10.1002 /$ pen. 10449

[2] Harkin-Jones E., Crawford R. J.: Rotational molding of liquid plastic systems: An assessment of material moldability. Advances in Polymer Technology, 15, 71100 (1996).

DOI: 10.1002/(SICI)1098-2329(199621)15:1<71::AIDADV6>3.0.CO;2-H

[3] Barhoumi N., Lamnawar K., Maazouz A., Jaziri M., Abdelhedi R.: Reactive rotational molding process of PP/PA6 bilayer systems: Experimental investigations. International Journal of Material Forming, 1, 671-674 (2008).

DOI: $10.1007 / \mathrm{s} 12289-008-0304-9$

[4] Mougin N., Veith C. A., Cohen R. E., Gnanou Y.: Anionic polymerization of lactams in the presence of metal dialkoxyaluminum hydrides: Presentation of a new mechanism. Macromolecules, 25, 2004-2016 (1992).

DOI: $10.1021 / \mathrm{ma} 00033 \mathrm{a} 026$

[5] Brouwer W. D., van Herpt E. C. F. C., Labordus M.: Vacuum injection moulding for large structural applications. Composites Part A: Applied Science and Manufacturing, 34, 551-558 (2003).

DOI: $10.1016 / \mathrm{S} 1359-835 \mathrm{X}(03) 00060-5$

[6] Macosko C. W.: RIM fundamentals of reaction injection molding. Hanser, Munich (1989).

[7] Rusu G., Rusu E.: Nylon 6/SiO 2 nanocomposites synthesized by in situ anionic polymerization. High Performance Polymers, 18, 355-375 (2006).

DOI: $10.1177 / 0954008306063392$

[8] Rusu G. H., Rusu M., Rusu E., Stoleriu A., Teaca C. A.: Direct centrifugal molding of nylon 6-based products from $\varepsilon$-caprolactam. Polymer Plastics Technology and Engineering, 3, 233-247 (2000).

DOI: $10.1081 /$ PPT-100100026

[9] Rusu G., Ueda K., Rusu E., Rusu M.: Polyamides from lactams by centrifugal molding via anionic ring-opening polymerization. Polymer, 42, 5669-5678 (2001). DOI: 10.1016/S0032-3861(01)00059-3

[10] Rudolf P., Vladimir K.: Lactam-based polyamides, Volume I: Polymerization structure. CRC Press, Boca Raton (1991).

[11] Goodman I., Kehayoglou A. H.: Anionic copolymers of caprolactam with laurolactam (nylon 6/12 copolymers) II. Crystallisation, glass transitions and tensile properties. European Polymer Journal, 19, 321-325 (1983).

DOI: $10.1016 / 0014-3057(83) 90167-2$
[12] Zhang C-L., Feng L., Hu G-H.: Anionic polymerization of lactams: A comparative study on various methods of measuring the conversion of $\varepsilon$-caprolactam to polyamide 6. Applied Polymer Science, 101, 1972-1981 (2006). DOI: 10.1002/app.23659

[13] Nelson W. E.: Nylon plastics technology. NewnesButterworths, London (1976).

[14] van Rijswijk K., Bersee H. E. N., Jager W. F., Picken S. J.: Optimisation of anionic polyamide- 6 for vacuum infusion of thermoplastic composites: Choice of activator and initiator. Composites Part A: Applied Science and Manufacturing, 37, 949-956 (2006).

DOI: 10.1016/j.compositesa.2005.01.023

[15] Khodabakhshi K., Gilbert M., Dickens P., Hague R.: Optimizing conditions for anionic polymerization of caprolactam for inkjetting. Advances in Polymer Technology, 29, 226-236 (2010).

DOI: $10.1002 / \mathrm{adv} .20191$

[16] Ueda K., Nakai M., Hosoda M., Tai K.: Synthesis of high molecular weight nylon 6 by anionic polymerization of $\varepsilon$-caprolactam. Mechanism and kinetics. Polymer, 29, 568-573 (1997).

[17] van Rijswijk K., Bersee H. E. N., Beukers A., Picken S. J., van Geenen A. A.: Optimisation of anionic polyamide- 6 for vacuum infusion of thermoplastic composites: Influence of polymerisation temperature on matrix properties. Polymer Testing, 25, 392-404 (2006). DOI: $10.1016 /$ j.polymertesting.2005.11.008

[18] Crawford R. J.: Rotational molding of plastics. John Wiley \& Sons, New York (1996).

[19] Luisier A., Bourban P-E., Månson J-A. E.: Time-temperature-transformation diagram for reactive processing of polyamide 12. Journal of Applied Polymer Science, 81, 963-972 (2001).

DOI: 10.1002/app.1518

[20] Udipi K., Davé R. S., Kruse R. L., Stebbins L. R.: Polyamides from lactams via anionic ring-opening polymerization: 1 . Chemistry and some recent findings. Polymer, 38, 927-938 (1997).

DOI: 10.1016/S0032-3861(96)00566-6

[21] Kim K. J., Hong D. S., Tripathy A. R.: Kinetics of adiabatic anionic copolymerization of $\varepsilon$-caprolactam in the presence of various activators. Journal of Applied Polymer Science, 66, 1195-1207 (1997).

DOI: $10.1002 /($ SICI) 1097-4628(19971107)66:6<1195 $\because$ AID-APP19>3.0.CO;2-0

[22] Crawford R. J., Throne J. L.: Rotational molding technology. Plastics Design Library, Norwich (2002).

[23] van Rijswijk K., Lindstedt S., Vlasveld D. P. N., Bersee H. E. N., Beukers A.: Reactive processing of anionic polyamide- 6 for application in fiber composites: A comparitive study with melt processed polyamides and nanocomposites. Polymer Testing, 25, 873-887 (2006). DOI: $\underline{10.1016 / \mathrm{j} . \text { polymertesting.2006.05.006 }}$ 
[24] Hakmé C., Stevenson I., Maazouz A., Cassagnau P., Boiteux G., Seytre G.: In situ monitoring of cyclic butylene terephtalate polymerization by dielectric sensing. Journal of Non-Crystalline Solids, 353, 4362 4365 (2007).

DOI: $10.1016 /$ j.jnoncrysol.2007.04.051

[25] Ricco L., Russo S., Orefice G., Riva F.: Anionic poly ( $\varepsilon$-caprolactam): Relationships among conditions of synthesis, chain regularity, reticular order, and polymorphism. Macromolecules, 32, 7726-7731 (1999). DOI: $10.1021 / \mathrm{ma9909004}$
[26] Young R. J., Lovell P. A.: Introduction to polymers. Chapman and Hall, London (1991).

[27] Throne J. L., Gianchandani J.: Reactive rotational molding. Polymer Engineering and Science, 20, 899-919 (1980).

DOI: 10.1002/pen.760201309

[28] Bellehumeur C. T., Kontopoulou M., Vlachopoulos J.: The role of viscoelasticity in polymer sintering. Rheologica Acta, 37, 270-278 (1998). DOI: $10.1007 / \mathrm{s} 003970050114$

[29] Liu S-J.: Sintering rheology of semi-crystalline polymers. International Polymer Processing, 13, 88-90 (1998). 